Sigmoidoscopy did not show any abnormality. A barium enema (Dr. A. Jenkins) revealed a fistulous communication between the colon, in the region of the hepatic flexure, and the gall-bladder. The opaque material passed along what was thought to be the common duct, where there was a filling defect which was believed to be a stone at the ampulla of Vater (see illustration).

Operation was carried out November 16, 1951, and the findings. were exactly as had been suggested radiologically.

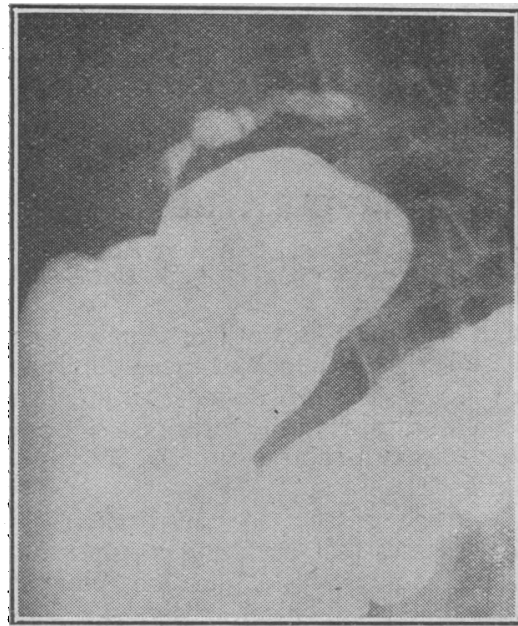

Radiograph showing the filling defect.
The hepatic flexure of the colon was adherent to the liver, and when the two organs were separated the gall-bladder was brought into view and was seen to communicate with the colon by a fistula. The fistula was divided and the opening in the transverse colon closed in layers. The junction of the cystic duct and the common bile duct was identified, and these ducts and the common hepatic duct were seen to be dilated. The pancreas felt normal. The common bile duct was explored and a stone was removed from the lower end. Another stone was found in the right hepatic duct. The ducts were washed out with saline, and, after the patency of the ampulla of Vater had been confirmed by means of a sound, the gall-bladder was removed and a drain placed over the common duct.

The patient made an uninterrupted recovery. The diarrhoea stopped immediately and has not recurred since.

R. H. Franklin, M.B., F.R.C.S. Department of Surgery, Postgraduate Medical School of London, W.12.

\section{Serum Sickness}

Antitetanus serum is given widely as a prophylactic measure in out-patient departments of general hospitals and by general practitioners throughout the country, and mild symptoms due to serum sickness are noted from time to time. The following case is interesting because of the splenic enlargement, a factor which may cause confusion in diagnosis from glandular fever and septicaemia. Splenomegaly has been reported in America, but apparently not in this country.

\section{CASE REPORT}

The patient, a girl aged 7 years, injured her left thumb so that the nail and pulp were hanging by a medial skin-flap. She was admitted to hospital on October 26,1951 , for suture under general anaesthesia, and was given a prophylactic dose of 1,500 international units of antitetanus serum subcutaneously into the right arm, and "distaquaine" penicillin 250,000 international units six-hourly. The wound healed and she remained very well and was discharged on October 31 . She returned to the out-patient department the next day, when the thumb was dressed, and appeared quite well. On November 2 , when she reported for further dressings, it was noticed that her temperature was $99.6^{\circ} \mathrm{F}$. $\left(37.6^{\circ} \mathrm{C}\right.$. $)$, and on examination tender enlarged, firm glands were found in both groins and popliteal fossae. The spleen was palpable two fingerbreadths below the left costal margin, but was not tender. There was no rash.

The patient was admitted to a medical ward, and on further examination four hours later was found to have palpable glands in both anterior cervical triangles and in the left axilla. There was an area of skin in the left hypochondrium 4 in. $(10 \mathrm{~cm}$.) in diameter, bright pink in hue, but not irritating. She had no temperature at this time. Her throat was normal, and she had no clinical jaundice. There was no apparent infection, and glandular fever was suspected. Blood examination showed: red cells, 4,530,000; haemoglobin, $78 \%$; leucocytes, 7,000 ; polymorphonuclears, $83 \%$; eosinophils $1 \%$; lymphocytes, $13.5 \%$; monocytes, $2.5 \%$. A Paul-Bunnell agglutination test was negative, as were the Widal and Brucella agglutination tests. The next day, November 3, the pink erythematous area on the trunk had disappeared, but the skin over the gland in the right groin was discoloured. No local infection was apparent. A vaginal swab was taken, which revealed no cause for the adenitis, and a course of penicillin was begun by injection into the right buttock.

On November 5 the glands were no longer tender, but the whole of the right buttock was red, though on palpation there was no tenderness. A few rubelliform spots were noticed on the abdomen. The penicillin was discontinued, the diagnosis of serum sickness having been made. On November 6 the buttock was normal in colour, but a rash was present on both legs. This consisted of circular lesions with pale centres encircled by red rings varying in size from 5 to $30 \mathrm{~mm}$. in diameter. The rash spread down her legs and faded lastly from her feet on November 8 .

The following tests were made on November 8 and 9 : Antitetanus serum was diluted to $1 / 100$ and $0.02 \mathrm{ml}$. was injected intradermally into the right forearm. Twenty minutes later a wheal measuring $12 \mathrm{~mm}$. in diameter and a flare measuring 24 by $30 \mathrm{~mm}$. were noted. Antitetanus serum was diluted $1 / 10$ and one drop was inserted into the left conjunctival sac : there was no reaction or irritation. (Claiborn in 1932 tested 465 patients with conjunctival and intradermal tests : $5.6 \%$ were positive to both tests ; $21.3 \%$ were positive to the intradermal test only. All patients positive to the conjunctival tests were also positive to the intradermal test.) Crystalline penicillin was diluted 1/100 and $0.02 \mathrm{ml}$. was injected intradermally into the left arm. Twenty minutes later there was no reaction.

Strazza (1946) reported delayed sensitization to penicillin similar to serum sickness. On a scratch test the reaction to penicillin 15,000 units per ml. was negative. Gordon (1946) reported three cases and reviewed the literature on reactions to penicillin simulating serum sickness. There was no reference to enlargement of the spleen, but one case had glandular enlargement. Scratch tests were sometimes positive.

Diphenhydramine hydrochloride was begun on November $9,20 \mathrm{mg}$. being given four-hourly. The glands in her neck, axilla, and popliteal fossae were no longer palpable on November 10. The glands in the groin had decreased in size and were not tender, and the spleen could just be felt on inspiration. On November 12 the spleen was no longer palpable.

Peterson and Bishop (1947) treated serum sickness with diphenhydramine hydrochloride, giving $50 \mathrm{mg}$. or more, up to $100 \mathrm{mg}$., six - to eight-hourly, depending on results. As relapse may follow withdrawal, they suggested carrying on for three days to a week, depending on the condition of the patient.

\section{COMMENT}

There was no history of asthma, urticaria, or hay-fever in the family, and this was apparently the first injection of horse serum the child had had. The first sign was a slight fever discovered seven days after the injection, though it is possible that the glands and spleen had enlarged before this time. The rash appeared on the tenth day, and varied in type over the next five days. The only complaint during these manifestations of serum sickness was tenderness in the groins.

I wish to thank Dr. H. R. E. Wallis for permission to publish this case and Dr. M. Marshall, house-surgeon, who transferred the child under my care.

MARY Whitfield, M.B., Ch.B.

REFERENCES

Claiborn, L. N. (1932). J. Amer. med. Ass., 98, 1718

Gordon, E. J. (1946). Ibid., 131, 727.

Peterson, J. C., and Bishop, L. K. (1947). Tbid., 133, 1277.

Strazza, J. A. (1946). Ibid., i30, 1071. 